\title{
Operation and Performance of the New Fermilab Booster $\mathrm{H}^{-}$Injection System
}

\author{
J. Lackey\#, F.G. Garcia, M. Popovic, E. Prebys \\ FNAL, Batavia, IL 60510, USA
}

\begin{abstract}
The operation and performance of the new, $15 \mathrm{~Hz}, \mathrm{H}^{-}$ charge exchange injection system for the FNAL Booster is described. The new system installed in 2006 was necessary to allow injection into the Booster at up to 15 $\mathrm{Hz}$. It was built using radiation hardened materials which will allow the Booster to reliably meet the high intensity and repetition rate requirements of the Fermilab's HEP program. The new design uses three orbit bump magnets (Orbumps) rather than the usual four and permits injection into the Booster without a septum magnet. Injection beam line modification and compensation for the quadrupole gradients of the Orbump magnets is discussed.
\end{abstract}

\section{INTRODUCTION}

In order to be able to inject at $15 \mathrm{~Hz}$ a design for a new Orbump magnet [1] intended to do a one-for-one replacement of the existing four magnet injection bump system had been completed. About the time magnet production had started a new injection design concept was proposed [2]. This design had several decided advantages. The required current for the new pulsed power supply was reduced to less than half, from $30 \mathrm{kA}$ to 13.4 kA and only three magnets were required. This design also completely eliminated the need for the 13 degree septum magnet which had proven to be a severe aperture limitation. This design, by maximizing the bend center spacing, would also minimize the vertical $\beta$ function distortion due to edge focusing of the Orbump magnets [3]. This was expected to yield a substantial increase in the vertical acceptance of the ring. This scheme would also permit implementation of phase space painting if desired that the previous method would not. The new design was carefully reviewed and then implemented with installation and commissioning occurring in 2006.

\section{INJECTION SYSTEM DESIGN}

The new system injects the beam at a relatively small angle, $\sim 70 \mathrm{mrad}$, to the injection closed orbit (In the previous system this angle was 13 degrees) through the center bump magnet (Orbump2). After exiting Orbump2 this angle has been reduced to $23.45 \mathrm{mrad}$. See Figure 1 .

*Work supported by the U.S. Department of Energy under Contract No. DE-AC02-76CH0300.

\#lackey@fnal.gov
This geometry allows the injected beam to miss the upstream gradient magnet by about $25 \mathrm{~mm}$. The stripping foil is positioned just downstream of the middle Orbump magnet. The separation of the bumped beam from the Booster closed orbit centerline is $\sim 45 \mathrm{~mm}$ at the foil position with the edge of the stripping foil positioned at $\sim 32 \mathrm{~mm}$.

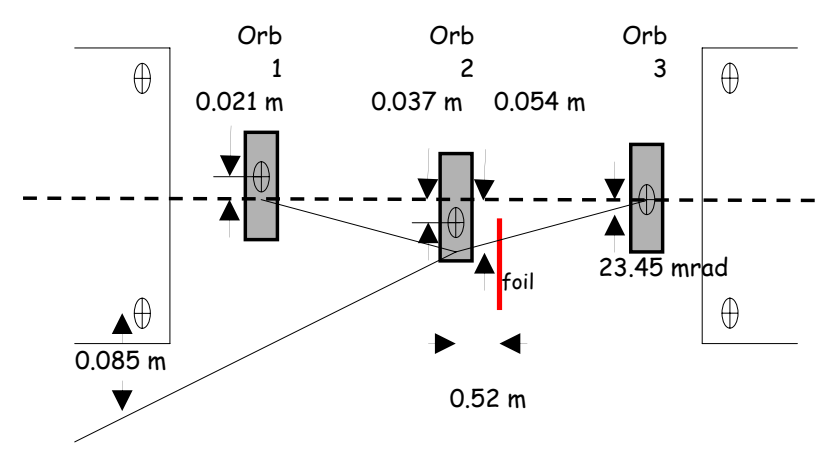

Figure 1

In this design the stripped electrons and the protons, stripped or un-stripped, are on same trajectory as they exit the stripping foil and do not separate until they enter the third bump magnet (Orbump3). The stripped electrons are deposited on the pole tip of this magnet. The protons exit Orbump3 on the nominal Booster closed orbit. $\mathrm{H}^{0}$ particles will continue un-deflected until they hit the magnet laminations on the radial outside of the second downstream gradient magnet. $\mathrm{H}^{-}$particles initially are deflected radially inward away from the closed orbit by Orbump3 but then are deflected radially outward across the closed orbit by the gradient magnet fields and are also deposited in the second gradient magnet. The deposition points of the $\mathrm{H}^{0}$ and $\mathrm{H}^{-}$beams are separated by about one meter on that magnet. The residual radiation levels at these points are routinely measured and data logged and both points are monitored with loss monitors as another means of assessing the condition of the foil.

Both the upstream and center Orbump magnets are offset horizontally. See Figure 1. The offsets were required to create the proper apertures and clearances for the injected and circulating beam. We took advantage of the offsets to cancel the effect of a small quadrupole gradient [1] in the Orbump magnet field. Adjusting the offsets and selecting the proper quad polarity (the polarity 
can be changed by turning the magnet end for end) allowed complete cancellation of the quadrupole error at injection. Though the field error is compensated during the multi-turn injection process a net dipole error is induced when the current in the Orbump magnets is ramped to zero after injection. Orbump magnets were selected based on field measurements to minimize the effect of the quad error and calculations indicated that this error would introduce a maximum orbit distortion of $\sim 0.7$ $\mathrm{mm}$ [4] with the set magnets chosen for installation.

A. Drozhdin simulated the new injection process with MAD and STRUCT [5]. This work looked at the effects on the Booster lattice due to changes to the injection system and from the removal of one of the two $8 \mathrm{Gev}$ extraction systems. MAD predicted a maximum orbit distortion of $\sim 2.5 \mathrm{~mm}$ during the current ramp-down. The reduction in the edge focusing effect lead to a predicted reduction $(23 \%)$ in distortion to the vertical $\beta$ function, from $\sim 27 \mathrm{~m}$ to $\sim 22 \mathrm{~m}$, the nominal design $\beta_{\max }$ being 20.3 $\mathrm{m}$. Little if any increase in emittance was seen in tracking studies from the growth and decay of the orbit distortion. This effect lasts for $\sim 50 \mathrm{uSec}$ or about 22 turns.

\section{INJECTION SYSTEM PERFORMANCE}

After installation and commissioning the only necessary modification to the system was improvement to the pulse forming network (PFN) of the Orbump pulsed power supply to increase the length of the pulse flattop and tuning of the PFN to reduce the current ripple. This work has led to a usable flattop of $44 \mu \mathrm{sec}$ and peak-topeak ripple of $\pm 0.29 \%$. This level of current ripple will induce a maximum position error during injection of \pm $0.145 \mathrm{~mm}$. This amount of motion error contributes negligibly to emittance growth. The $44 \mu \mathrm{sec}$ flattop will allow injecting over 20 revolutions of beam into the Booster.

The study by Drozhdin [5] looked extensively at emittance growth due to errors in the matching of the beam position, angle, and mismatch of the various lattice parameters. The optics of the downstream end of the $400 \mathrm{MeV}$ transport line to Booster were redesigned to handle the new simplified 3-bump injection scheme. Most of the design effort went into producing a better matching to the modified Booster lattice as predicted by MAD [5]. The transfer line optics measurements were obtained using differential orbit techniques which helped the development of a detailed optics model of the line. The measured beam size and dispersion functions are in good agreement with the calculation result at each monitor in the transport line. To illustrate this, Figure 2 shows the rms transverse beam size for both the prediction and the real measurement. Actually, many small but steady advances made on the transfer line optics paved the way to a better injection into the Booster machine. In order to aid the injection efficiency into Booster tuning knobs are implemented which allow modifying the lattice matching parameters over a range of $\pm 20 \%$.
Early-on an unexpected beam loss point was noted just downstream of the stripping foil. It was quickly realized that this loss was due to the multiwire beam profile monitors in the injection beam line stripping some of the injected beam. The stripped particles, both $\mathrm{H}^{0}$ and $\mathrm{H}^{+}$, will hit the beam pipe immediately after the foil.

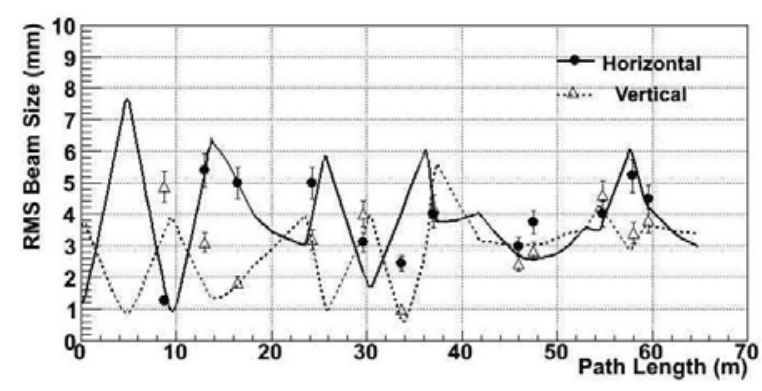

Figure 2: The rms beam sizes vs. beam line path length. The curves illustrate the theoretical rms beam sizes. Comparison to the measured horizontal (filled circle) and vertical (open triangle) are also shown.

Residual radiation levels at this point remain near 15 to 20 $\mathrm{mRem} /$ hour since periodic use of the multiwires to monitor beam parameters is necessary. All other monitored points on the injection girder have been reduced from 100 to $200 \mathrm{mRem} / \mathrm{hr}$ to typically less than $10 \mathrm{mRem} / \mathrm{hr}$.

\section{MODIFICATIONS AND CHANGES}

We are going to install a single wire scanner downstream of the injection girder between the first two gradient magnets that will be able to look at the $\mathrm{H}^{0}, \mathrm{H}+$ and $\mathrm{H}^{-}$beams. At this point the beams have separated enough to do this. This will permit additional monitoring and testing of the condition of the stripping foil and should allow stripping efficiency measurements of the foils themselves.

Some slight mechanical modifications to the girder are required to permit installation of a new corrector magnet during the upcoming shutdown. There will still be room on the girder downstream of the stripping foil for the addition of a dedicated electron catcher although the bpm in the new corrector magnet is un-terminated so that a bias voltage can be applied to catch the stripped electrons.

\section{ACKNOWLEDGMENTS}

The new injection system for the Booster works very well indeed. It is responsible in part for the ever increasing Booster acceleration efficiency and copious numbers of protons that the Proton Source is providing for Fermilab's experimental program. We are indebted to many people in the Accelerator Division and Technical 
Division for their hard work in designing, building, installation and commissioning the new system.

\section{References}

[1] J.R. Lackey, J.A. Carson, C.M. Ginsburg, H.D. Glass, D.J. Harding, V.S. Kashikhin, A. Makarov, E.J. Prebys, "New Pulsed Orbit Bump Magnets For The Fermilab Booster Synchrotron" PAC 2005.

[2] M. Popovic, C. Ankenbrandt, F. G. Garcia, J. Lackey, W. Pellico and E. Prebys, "A Proposed $\mathrm{H}^{-}$Injection System for the Fermilab Booster", FNAL beams-doc-1784-v1.

[3] W. Chou, A. Drozhdin, P. Lucas, F. Ostiguy, "Booster Modeling and Space Charge Study", FNAL beams-doc-632-v1.

[4] E. Prebys, “Optimum Booster ORBUMP Magnet Configuration”, FNAL beams-doc-2112-v2.

[5] A. Drozhdin, "Study of a Proposed Injection and new extraction Systems for the Fermilab Booster", http://www-ap.fnal.gov/ drozhdin/prdriver/ 